\title{
Review Article \\ Detection and Prognosis of Prostate Cancer Using Blood-Based Biomarkers
}

\author{
Wei Jin $\mathbb{D}^{1},{ }^{1}$ Xiang Fei, ${ }^{1}$ Xia Wang, ${ }^{1}$ Yan Song $\mathbb{D}^{1},{ }^{1}$ and Fangjie Chen $\mathbb{D}^{2}$ \\ ${ }^{1}$ Department of Urology, Shengjing Hospital of China Medical University, Shenyang, Liaoning, China \\ ${ }^{2}$ Department of Medical Genetics, School of Life Sciences, China Medical University, Shenyang, Liaoning, China
}

Correspondence should be addressed to Fangjie Chen; chenfj@cmu.edu.cn

Received 28 February 2020; Revised 24 March 2020; Accepted 27 April 2020; Published 4 May 2020

Guest Editor: Xiaolu Jin

Copyright (C) 2020 Wei Jin et al. This is an open access article distributed under the Creative Commons Attribution License, which permits unrestricted use, distribution, and reproduction in any medium, provided the original work is properly cited.

\begin{abstract}
Prostate cancer (PCa) is second only to lung cancer as a cause of death. Clinical assessment of patients and treatment efficiency therefore depend on the disease being diagnosed as early as possible. However, due to issues regarding the use of prostatespecific antigen (PSA) for screening purposes, PCa management is among the most contentious of healthcare matters. PSA screening is problematic primarily because of diagnosis difficulties and the high rate of false-positive biopsies. Novel PCa biomarkers, such as the Prostate Health Index (PHI) and the 4 Kscore, have been proposed in recent times to improve PSA prediction accuracy and have shown higher performance by preventing redundant biopsies. The 4Kscore also shows high precision in determining the risk of developing high-grade PCa, whereas elevated PHI levels suggest that the tumor is aggressive. Some evidence also supports the effectiveness of miRNAs as biomarkers for distinguishing PCa from benign prostatic hyperplasia and for assessing the aggressiveness of the disease. A number of miRNAs that possibly act as tumor inhibitors or oncogenes are impaired in PCa. These new biomarkers are comprehensively reviewed in the present study in terms of their potential use in diagnosing and treating PCa.
\end{abstract}

\section{Overview}

Prostate cancer (PCa), which manifests as solid tumors, is the most prevalent form of cancer in male individuals. PCa is second only to lung neoplasms in terms of mortality rate, and its incidence rises with age in numerous countries. In the US alone, around 2.8 million male individuals are diagnosed annually with PCa [1]. A marked increase has also been noted recently in the incidence of PCa in China due to factors such as lifestyle changes and more efficient diagnosis. The incidence of PCa may also differ due to different dietary habits. Dietary factors, like the consumption of protein, fat, carbohydrates, vitamins, and polyphenols, may have an important role in PCa pathogenesis [2, 3]. Adequate diet and physical activity trigger alterations in the serum factors that retard the development of androgen-reliant PCa cells and cause them to die $[2,4]$. Conversely, PCa can be promoted by a high body mass index, hypertension, and a number of metabolic factors $[5,6]$. PCa is characterized by a high degree of inhomogeneity, as it can manifest as either latent forms or highly aggressive forms that lead to morbidity and death due to metastasis to other parts of the body [7-9].

PCa screening is based on the use of the prostate-specific antigen (PSA), a feature that has made this disease notably contentious within the healthcare field. PCa exhibited a staggering rise in incidence during the period from 1986-1991, when the PSA test first became available. However, subsequent prostate biopsies frequently revealed no correlation between elevated PSA levels and the presence of cancer in many cases. Indeed, PSA levels do not exceed $4.0 \mu \mathrm{g} / \mathrm{L}$ in $20-25 \%$ of the cases with a PCa diagnosis [10]. The results of cytology or histopathology are the basis of a confirmed PCa diagnosis, especially in cases where the PSA levels lie between 4 and $10 \mu \mathrm{g} / \mathrm{L}$ - the so-called "grey area." By contrast, about one-quarter of the cases with PSA levels of $2-10 \mu \mathrm{g} / \mathrm{L}$ has positive biopsies.

PSA is not an exclusive marker of cancer because it is produced by cancerous prostate cells and by healthy prostate cells. Therefore, factors such as a large prostate size, benign prostatic hyperplasia $(\mathrm{BPH})$, or prostatitis can trigger false 
positives and result in an incorrect PCa diagnosis and treatment [11]. The United States Preventive Services Task Force issued a recommendation in 2018 that PCa screening should not be performed on the basis of PSA levels in cases of male individuals refusing the procedure or in male individuals 70 years of age or older [12]. Furthermore, unimportant tumors may be excessively detected due to PSA-based screening, leading to unnecessary treatment. In addition, the possible positive outcomes of screening are no greater than the adverse outcomes of excessive detection and unnecessary treatment [13]. Diagnosis of PCa based only on PSA levels is therefore gradually losing its appeal as a clinical approach [14]; however, this has not put an end to the controversy surrounding screening $[15,16]$. A recent study suggested that although discontinuation of PSA-based screening would eliminate excessive diagnoses, the lack of screening would lead to a complete failure to avoid preventable deaths and would increase the PCa-related mortality rate by 13 $20 \%$ [17].

In recent times, various initiatives have been launched to identify novel subtypes of PSA that could make PSA-based screening more precise for diagnosing early PCa and formulating a prognosis for it. These initiatives have yielded several novel PCa biomarkers, including the PHI, a biomarker that has received the approval of the US Food and Drug Administration (FDA) for prostate cancer detection [18]. Another PCa biomarker is the 4 Kscore, otherwise known as the 4kallikrein panel, which screens for total PSA, fPSA, intact PSA (iPSA), and human kallikrein 2. MicroRNAs are another source of promising PCa biomarkers that have emerged as potential alternatives to PSA, owing to the progress being made in deep sequencing technology [19]. Taking all these aspects into account, the aim of the present study is to review the new understanding that has been achieved regarding $\mathrm{PCa}$ blood-based biomarkers and the role that they can play in diagnosing and treating early PCa.

\section{The Prostate Health Index (PHI)}

The importance of proPSA as a component of IPSA was highlighted in 1997 by the revelation that the serum of individuals with PCa contained truncated proPSA forms [20]. Preliminary findings confirmed that PCa could be detected based on proPSA isoforms, leading to fewer negative biopsies in cases with PSA levels in the "grey area." A reliable immunoassay has since been made available on the market by Beckman Coulter to assess [-2] proPSA, and this immunoassay has been extensively investigated for its relevance for use in managing early PCa. Overall, PCa patients have a notably higher ratio of [-2] proPSA to PPSA (\%p2PSA).

The [-2] proPSA immunoassay has now been integrated alongside fPSA and tPSA in the PHI test. This test is highly specific for $\mathrm{PCa}$ of clinical importance and has proven effective in detecting this disease $[21,22]$. It enables differentiation between benign prostatic hyperplasia $(\mathrm{BPH})$ and $\mathrm{PCa}$ in suspected cases, but it can also enhance the accuracy of detection of high-grade cancer, thereby preventing redundant biopsies. In June 2012, this biomarker received FDA approval for use in detecting PCa in male individuals 50 years of age or older who had PSA levels of $4-10 \mu \mathrm{g} / \mathrm{L}$ and had undergone a digital rectal examination (DRE) without abnormal outcomes. The National Comprehensive Cancer Network also endorses the PHI test in cases where a biopsy has not been conducted or has produced negative results, and the Network specifies that a significant risk of PCa is reflected by PHI results higher than 35 [23].

Prospective research conducted in multiple centers among a sample of 2499 men has indicated that the use of PHI values higher than 30 at $90 \%$ sensitivity for high-grade PCa (HGPC) (Gleason $\geq 7$ ) could have prevented Gleason 6 PCa diagnoses in 33\% of the cases and biopsies in 56\% of the cases of Asian men. Similarly, the use of PHI higher than 40 at $90 \%$ HGPC sensitivity could have prevented Gleason 6 PCa diagnoses in $31 \%$ of the cases and biopsies in $40 \%$ of the cases of Caucasian men $[24,25]$. Prediction of biopsy results based on PHI and the \%p2PSA score has now been advocated by a number of researchers. The usefulness of these measurements in cases where the PSA levels fall between 2 and $10 \mu \mathrm{g} / \mathrm{L}$ has been demonstrated by two studies conducted in multiple centers in 1362 and 646 patients. The \%p2PSA was associated with an area under the curve (AUC) value of 0.72 , while the PHI was associated with an AUC value of 0.74 [23]. Both biomarkers considered together were associated with an AUC value of 0.67 [26]. Chiu et al. suggested that PHI was effective in cancer risk stratification for both European and Asian subjects [25]. However, populationspecific PHI reference ranges were different and should be used [25]. These findings support the relevance of the PHI test for identifying cases among Asian and Caucasian individuals with a high risk of $\mathrm{PCa}$ and thereby minimizing redundant biopsies and excessive PCa diagnosis.

When compared with the total PSA and \%fPSA, the combination of \%p2PSA and PHI provided a greater precision of PCa detection at biopsy $[23,27]$. Furthermore, the two biomarkers were well-correlated with tumor aggressiveness, occurring at higher levels in cases of Gleason $>6$. These findings have been confirmed by two meta-analyses from 2013 and 2014, which indicated that \%p2PSA and PHI were, respectively, associated with AUCs of $0.635-0.78$ and $0.69-$ $0.781[28,29]$. These meta-analyses additionally highlighted the superior outcomes obtained with these two biomarkers compared with those obtained with PSA and \%fPSA. The latter observation was reinforced by a later meta-analysis, which undertook a comparison between PHI and \%fPSA in cases with total PSA levels of $2-10 \mu \mathrm{g} / \mathrm{L}$. In that meta-analysis, PHI had an AUC value of 0.74 , while \%fPSA had an AUC value of 0.63 [30].

Some researchers have argued that PCa could be predicted clinically with greater accuracy by incorporating PHI and \%p2PSA into multivariate models that take into account the integration of PSA and a range of clinical and demographic factors. For instance, the incorporation of PHI and $\% 2$ pPSA into a multivariate model based on patient age, prostate volume, PSA, and \%fPSA was reported to increase the AUC value from 0.762 to 0.802 or 0.815 , depending, respectively, on whether a logistic regression analysis or an artificial neural network was applied [31]. Similarly, a different study on 112 patients with Gleason 6 who underwent 
radical prostatectomy reported that the patients with a final histology upgrade to Gleason 7 or higher exhibited markedly increased PHI values [32]. Another study found that PHI had an AUC value of 0.815 for the detection of GHPC in cases of Gleason 7 or higher [33] and suggested that the ideal PHI cut-off point of 24 at 95\% sensitivity for detection of aggressive PCa. The use of this cut-off point prevented redundant biopsies in $41 \%$ of the cases. By contrast, an active monitoring program involving 167 patients revealed that the baseline and longitudinal \%p2PSA and PHI levels allowed better prediction of renewed biopsy categorization at follow-up, whereas total PSA level showed no similar correlation with renewed biopsy categorization [34].

\section{The 4Kscore}

Another test that can predict the likelihood of occurrence of high-risk $\mathrm{PCa}$ is the 4Kscore or 4-kallikrein panel. The 4Kscore consists of four kallikrein blood markers-total PSA, free PSA (fPSA), intact PSA (iPSA), and the human kallikrein-related peptide 2 (hK2) - and has received ample research attention. For example, researchers from the Memorial Sloan-Kettering Cancer Center have found an association between the 4 Kscore and AUC values that exceeded not only the AUC values associated with a PSA-based model for the detection of all types of PCa (AUCs $=0.674-0.832$ ) but also the AUC values for HGPC detection with Gleason of 7 or higher (AUCs $=0.793-0.870) \quad[35,36]$. A comparison of the equivalent clinical models for high-risk PCa prediction yielded similar results. The model incorporating the variables of PSA, patient age, and DRE had AUC values of 0.7090.868 , whereas the addition of PPSA, iPSA, and hK2 to the model led to AUC values of 0.798-0.903 [37].

One major factor that $\mathrm{PCa}$ risk predictions typically take into account is prostate volume, as this factor has an impact on the levels of PSA in the serum. However, one study reported that the method used to perform the 4 Kscore to detect PCa was unaffected by the prostate volume [38]. This was the conclusion reached from a biomarker investigation in two groups of patients ( $n=2914, n=740$, respectively) with PSA levels of $3 \mu \mathrm{g} / \mathrm{L}$ or higher. The inclusion of the prostate volume in a model underpinned by 4 Kscore, patient age, and DRE led to an increase in AUC from 0.856 to 0.860 in the first group, whereas the inclusion or exclusion of the prostate volume made no difference to the AUC value in the second group (0.802).

The 4Kscore was made available on the market by Opko Diagnostics. It constitutes an algorithm that generates predictions about HGPC by integrating the 4-kallikrein panel with patient age, DRE, and history of previous biopsy. The US Food and Drug Administration FDA has not yet approved this test, but the National Comprehensive Cancer Network issued a recommendation in June 2015 that the 4 Kscore could be employed to screen for HGPC in cases where biopsy had either not been conducted or where it had produced negative results $[39,40]$.

In a prospective study conducted in 26 urology centers throughout the US, the 4Kscore achieved an AUC value of 0.82 when it was used to assess 1012 male patients about to undergo a prostate biopsy. The use of a $6 \%$ cut-off point was found to eliminate the necessity of a biopsy in $30 \%$ of the cases and deferred diagnosis in only $1.3 \%$ of the HGPC cases [41]. By contrast, a different study reported that the 4Kscore enabled the prediction of HGPC in cases with PSA levels exceeding $10 \mu \mathrm{g} / \mathrm{L}$ or with a positive DRE [42]. This ability of the 4Kscore to decrease the number of biopsies without overlooking a significant proportion of HGPCs has been highlighted by a number of studies $[43,44]$. The biopsy was deemed unnecessary in approximately half of the 262 cases scheduled for prostate biopsy because of PSA levels of $3 \mu \mathrm{g} / \mathrm{L}$ or higher, but the diagnosis of aggressive PCa was overlooked in just $1 \%$ of these cases when the 4 Kscore was used [45]. A different study on a sample of 740 male individuals who had been referred for biopsy due to high PSA levels reported comparable results [46].

The efficacy of the 4 Kscore for predictions was demonstrated by a study initiated in 1986 in Sweden on a sample of patients of different ages (40,50, and 60 years old) [47]. The patients were divided into two groups based on their 4Kscore outcomes at 50 and 60 years of age to determine how likely they were to develop remote metastasis after two decades. The study concluded that remote metastasis was more likely to develop in patients with a 4 Kscore exceeding 5 at 50 years of age and with PSA levels of $2 \mu \mathrm{g} / \mathrm{L}$ or higher, as well as in patients with a 4 Kscore exceeding 7.5 at 60 years of age and PSA levels of $3 \mu \mathrm{g} / \mathrm{L}$ or higher. Biopsy was considered unnecessary in cases that had moderate increases in PSA levels during midlife and a low risk of HGPC based on the 4Kscore. A different study on 1423 PCa patients showed that remote metastasis occurred in 235 patients with PSA levels exceeding $2 \mu \mathrm{g} / \mathrm{L}$. However, metastasis was much better predicted by a prespecified model underpinned by the four kallikrein markers than by PSA alone [47]. On the whole, the available research results support the efficiency of the 4 Kscore for use in early PCa diagnosis and prognosis.

One study compared the efficacy of the 4Kscore and PHI in a sample of 531 male individuals with PSA levels of 3$15 \mu \mathrm{g} / \mathrm{L}$. Neither biomarker differed markedly in the detection of either any-grade PCa or HGPC. The 4Kscore had AUC values of 0.69 and 0.718 , while the PHI had AUC values of 0.704 and 0.711 [48].

\section{The Usefulness of MicroRNAs as PCa Biomarkers}

4.1. miRNAs and PCa. Ample research has recently explored the function of protein-coding genes in oncogenesis. Transcription of the human genome to messenger RNA (mRNA) occurs in a proportion of $90 \%$, but protein encoding is undertaken by just $2 \%$ of the genome. Therefore, many RNAs do not encode for any protein. According to the findings from the latest studies, a genome segment without protein encoding is involved in oncogenesis $[49,50]$.

The expression of genes is regulated by endogenously expressed small, noncoding, single-stranded RNAs known as microRNA (miRNAs). Adverse regulation of gene expression by miRNA genes occurs after transcription, with the miRNA genes attaching primarily to the $3^{\prime}$ untranslated 
region $\left(3^{\prime}\right.$-UTR) of the coding gene, thereby suppressing mRNA translation. In the case of PCa, this suppression also promotes pathways for $\mathrm{PCa}$ progression by triggering translational repression or mRNA deterioration $[49,50]$. The types of miRNA complexes present in human plasma and serum were investigated in one study by differential centrifugation and size-exclusion chromatography of the miRNAs [51]. Most of the miRNAs were found in the plasma and serum in a form that did not undergo membrane binding, whereas a few were vesicle-associated miRNAs. A different study suggested that miRNAs from human serum, saliva, and other biological fluids could be made more sensitive through exosome isolation [52].

The physiological and pathological processes showing miRNA involvement include development, differentiation, metabolism, immunity, proliferation, apoptosis, senescence, cell identity, and stem cell maintenance [50,53]. A number of miRNAs that participate in the development of human cancer have been identified, and these appear to act as oncogenic factors that prevent the activity of tumor-suppressing genes or as tumor-suppressing miRNAs that activate oncogenes [54].

Novel biomarkers for the diagnosis and prognosis of cancer can be discovered by investigating cancer-related abnormal miRNA expression profiles. Many methods have been used to detect miRNAs, including northern blotting, nextgeneration sequencing (or RNA sequencing, RNA-Seq), microarrays analysis, reverse transcription PCR (RT-PCR), highly sensitive biosensors, and computational prediction [55]. In 2008, the first circulating miRNA profile of the serum of patients with diffuse large B-cell lymphoma was published as an aid for cancer diagnosis [56]. In many human cancers, the processes of development, invasion, and progression occur through direct involvement of hundreds of miRNAs with modified expression, and the cancer-related genomic area contains approximately half of the miRNA genes. PCa development is promoted by this type of dysfunctional regulation through the upregulation of oncogene modulation or downregulation of tumor inhibitors [50].

The current findings indicate the notable feature that the classification of human cancer origins can be based on miRNA signatures [57]. Thus, metastatic cancers of unknown origin can be effectively identified with high tissue specificity based on their miRNAs. For instance, a study of 208 tumors consisting of 15 distinct histologies, including $\mathrm{PCa}$, revealed successfully and estimated the primary tumor origin by profiling miRNA expression in paraffinembedded tissue [58]. A novel algorithm formulated in that study also categorized the miRNAs with $85 \%$ general precision (CI: 79-89\%). This algorithm was successful in determining the primary origin of 42 of 48 metastases ( $88 \%$ precision; CI: 75-94\%). However, these results must be verified by further research.

Several studies have highlighted an association between $\mathrm{PCa}$ and the modifications observed in the expression of miRNAs, which are associated with dysfunctional cell proliferation, differentiation, progression, and other processes [59, 60]. Comprehending the mechanism by which miRNAs interact with their targets and the implications of those inter- actions for PCa development is important for identifying the central part played by miRNAs in PCa [61]. This comprehension can also help in developing efficient treatment interventions, especially because aberrant miRNA expression is now viewed as a useful biomarker for diagnosing and classifying $\mathrm{PCa}$ and for determining its prognosis [62].

4.2. Oncogenic miRNAs and PCa. Cancer cell progression is promoted by miRNAs that serve as oncogenic miRNAs [63]. Calin and colleagues [64] were the first to investigate the correlation between miRNA dysregulation and cancer. Specifically, the authors observed that the dysregulation of miR-15 and miR-16 contributed to the development of chronic lymphocytic leukemia. PCa is typically associated with overexpression of miRNAs (e.g., miR-21, miR-32, miR-221, miR-222, miR-181, miR-18a, and miR-429) that have a crucial function in the regulation of PI3K/AKT, the epithelial-to-mesenchymal transition (EMT), cell proliferation, apoptosis, and androgen receptor (AR) expression $[65,66]$.

The androgen-regulated oncogenic miRNAs with expression in PCa include miR-21 and miR-32 [67]. MiR-21 regulates the expression of a large number of mRNA targets associated with microvascular proliferation and tumor invasiveness, and its expression is correlated with weak biochemical recurrence-free survival. Therefore, miR-21 expression can be used to estimate the probability of biochemical recurrence in PCa patients who have undergone radical prostatectomy [68]. The expression of miR-21 is also associated with castration resistance and metastasis, and its level increases linearly with clinical parameters such as the Gleason score and lymph node metastasis [59]. A study conducted on patients who had undergone radical prostatectomy revealed a close correlation between elevated miR-21 levels and advanced disease staging, lymph node metastasis, and poor patient outcomes. A multivariate analysis revealed that miR-21 expression was an independent estimator of biochemical recurrence after five years [69]. Hence, miR-21 can be used as a biomarker that anticipates cancer progression [70].

An additional miRNA with androgen regulation (via dihydrotestosterone [DHT] stimulation) is miR-32. This miRNA has a higher expression in castration-resistant prostate cancer (CRPC) cases than in benign prostatic cases. Its oncogenic activity includes targeting B-cell translocation gene 2 (BTG-2) as well as phosphoinositide-3-kinase interacting protein 1 (PIK3IP1). This miRNA suppresses apoptosis and encourages cells to proliferate by stimulating cell growth, as revealed by observations of miR-32 effects in the LNCaP PCa cell line. On the whole, miR-32 manifests its oncogenic activity by targeting the tumor-suppressing genes that control the ability of cells to proliferate, survive, and migrate $[71,72]$.

The oncogenic reprogramming of PCa-mobilized stem cells is believed to occur with the involvement of oncogenic miR-125b, miR-130b, and miR-155 [73, 74]. Mechanistic studies confirm that stem cell neoplastic reprogramming is likely triggered by miR-125b and miR-155 exosomal trafficking via targeted suppression of the sizable tumor- 
suppressor homolog2 and programmed cell death by the neoplastic transformation suppressor protein 4 [75]. Furthermore, miR-125 has been observed to target apoptosispromoting genes in $\mathrm{PCa}$, thus functioning as an oncogene [76]. Apoptosis is suppressed and cell proliferation is intensified when miR-125b, a miRNA triggered by an androgen receptor, is activated in the LNCaP cells. Some evidence supports miR-125b targeting of the mediator of Mdm2 sequestration, $\mathrm{p} 14^{\mathrm{ARF}}$ and subsequent disruption of $\mathrm{Mdm} 2$ deterioration, and triggering of the p53 network [77].

The expression of $\mathrm{p} 27^{\mathrm{Kip} 1}$, which regulates the cell cycle, is susceptible to the effects of several miRNAs, such as miR221, miR-222, and miR-429 [78]. Two of these, miR-221 and miR-222, are encoded on the $\mathrm{X}$ chromosome and share a seed sequence. Both of these miRNAs manifest their oncogenic effects by targeting a number of tumor-suppressing genes, and they both display overexpression in PCa. Abnormal expression of these two miRNAs can aid in prognosis because this expression has a close association with metastatic CRPC [78].

A modified miR-9 signature has been correlated with the progression and metastasis of PCa [79]. Similarly, miR-27a targets prohibition in $\mathrm{PCa}$, thereby regulating $\mathrm{AR}$ processing [61]. PCa tissues and cell lines also show high expression of miR-18, which targets the tumor-suppressing serine/threonine-protein kinase 4 , thereby functioning as an oncogenic miRNA [80]. One study showed that the inhibition of miR18a slowed PCa cell and tumor growth by promoting apoptosis triggered by Akt dephosphorylation with STK4 mediation [80]. These findings support the use of the peripheral blood oncogenic miR-18a as a noninvasive PCa biomarker and for differentiating $\mathrm{PCa}$ from $\mathrm{BPH}$ [81].

Important stages in PCa progression are associated with poor prognosis; these include bone metastasis and events affecting the skeleton [82]. Extensive research has been conducted on the involvement of miRNAs in cancer metastasis. PCa has been associated with the upregulation of miR-96, miR-154, and miR-409-5p, which are likely very important promoters of cancer colonization of bone during metastasis [83-86]. Additional evidence produced by targeting major fibroblast-activating molecules has shown a correlation between PCa progression and increased expression of miR133b, miR-409, and miR-210 [84, 85].

4.3. Tumor-Suppressing miRNAs and PCa. Tumor-suppressing gene action may be an additional function of miRNAs. Cancer cells generally exhibit downregulation of tumorsuppressing miRNAs, thereby promoting proliferation [87], the epithelial-mesenchymal transition (EMT), invasiveness, and metastasis. PCa progression could be monitored by evaluating the expression pattern of tumor-suppressing miRNA as a biomarker, and the same pattern could also be targeted by various treatment interventions [88].

Reduced expression of miRNA has been associated with cancer progression in a number of studies. PCa with castration resistance can develop through priming of $\mathrm{AR}$ and AR-V7 upregulation with hnRNPH1 mediation following dysregulation of miR-212 [89]. The PCa tumor promoter EGFR is targeted by miR-133 and miR-146a, thereby pre- venting disease progression. Therefore, intensified EGFR signaling may explain the loss of these two biomarkers and the subsequent aggressive progression of PCa [90]. Evidence has also been produced to indicate that the LNCaP, DU145, and PC3 PCa cell lines, as well as PCa tissues, exhibit reduced expression of miR-335 [91]. Another miRNA that inhibits tumors is miRNA-30a, a miRNA that targets sine oculis homeobox homolog 1 to prevent cancerous cells from proliferating and invading other tissues [92].

$\mathrm{PCa}$ is also associated with the downregulation of miR145, miR-200, miR-29b, miR-205, and miR-940, which leads to the upregulation of genes associated with the EMT. During the EMT, cells lose their attachment to other cells, and epithelial cells acquire the abilities of migration and invasion through conversion to mesenchymal stem cells capable of differentiation into various other cell types. Reduced expression of miR-29b and miR-130b in PCa is also correlated with extracellular matrix regulation through the targeting of matrix metalloproteinase 2 (MMP2) $[93,94]$. EMT and cancer invasion are inhibited by miR-200 members through a direct suppression of the zinc-finger E-box binding homeobox 1 and 2 (ZEB1 and ZEB2) translation factors [95]. Both prostate tumors and cancer cell lines have been associated with miR-200b downregulation, and miR-200b expression contributes significantly to the regulation of tumor invasion, metastasis, and chemosensitivity [96]. Enhanced expression of miR-200b targets Bmi-1, thereby preventing the proliferation and migration of PCa cells, as well as increasing PCa cell chemosensitivity to docetaxel.

In general, hindering the EMT by miR-200c appears to support the maintenance of the "epithelialness" of cancer cells $[97,98]$. According to one study, PCa was prevented from progressing by miR-205 downregulation that targets laminin-332, integrin- $\beta 4$, and MMP- 2 to regulate the extracellular matrix [99]. In addition, miR-205 targets the Bcl-2 protein to suppress apoptosis. The loss of miR-205 is significant in androgen-autonomous expression equivalent to Bcl-2 upregulation and possibly in reduced sensitivity to docetaxel in PCa [100].

An association has been established between decreased expression of miR-146a in PCa and angiogenesis suppression through targeting of the epidermal growth factor receptor pathway [101]. The progression and metastasis of PCa are stimulated by miR-1 downregulation through targeting of Src and TWIST1 [102]. EMT regulation and PCa progression are also mediated by targeted downregulation through the loss of miR-154 due to stromal antigen 2 (STAG2), the loss of miR-203 due to tumor growth factor-alpha (TGF- $\alpha$ ), and the loss of miR-224 due to Tribbles Pseudokinase 1 (TRIB1) $[103,104]$.

The original members of the family of tumor-suppressing miRNAs are miR-15a and miR-16. These two miRNAs are in charge of regulating the expression of numerous oncogenes, such as BCL2, MCL1, CCND1, and WNT3A. The effects of miR-15 and miR-16-1 on the BCL2 gene product trigger apoptosis [105-107]. By contrast, the loss of these two miRNAs elevates the levels of cyclin D1, an important regulator of the transition from the $\mathrm{G} 1$ to the $S$ phase. The loss of these two miRNAs also activates the WNT3a gene and the 
precancerous Wnt signaling pathway. Notably, cancer progression is stimulated by the cooperation between increased miR-21 expression and the loss of miR-15 and miR-16, as revealed by mouse models of $\mathrm{PCa}$. One study found that some PCa patients exhibited deregulated miR-15/miR-16 and $\mathrm{miR}-21$, and this deregulation was related to suboptimal prognosis and disease-free survival [108].

The expression and activity of the AR gene also seem to be targeted by a number of tumor-suppressing miRNAs. Proliferation is inhibited and apoptosis intensified by miR-488* [109], which can successfully reduce the expression of the AR protein in androgen-responsive as well as androgenrefractory PCa cells. PCa cells that overexpress miR-488* have also been shown to have lower PSA expression. Similarly, the direct downregulation of AR expression by excessive expression of $\mathrm{miR}-488 *$ in $\mathrm{PCa}$ cells can prevent cell growth and enhance apoptosis [109].

AR expression is significantly affected by miRNAs like miR-125b, miR-135a, and miR-27a [76, 110]. Androgenresponsive PCa cells display the upregulation of miR-135a, which therefore seems to have a tumor-inhibiting effect. An androgen-responsive component is also demonstrated by $\mathrm{miR}-125 \mathrm{~b}$ in the gene promoter area. Research on castrated mice revealed that the upregulation of miR-125b caused prostate tumor xenografts to exhibit androgenindependent growth, suggesting that the miRNA suppressed apoptosis [110].

AR expression regulates the expression of miR-27a as well. The expression of prohibitin, a tumor-suppressing gene, and AR corepressor, is downregulated when miR-27a expression is increased through androgen mediation. By facilitating a reduction in the expression of the prohibitin protein product, miR-27a upregulates the AR target gene expression and enhances the growth of PCa cells [111]. On the whole, the wide range of functions fulfilled by miRNAs in PCa development has received substantial attention in the search to understand how miRNAs aid and inhibit the disease process.

4.4. Exosomal miRNAs. The shortcomings of the existing protocols for PCa screening call for the discovery of novel biomarkers. Effective management of this disease and improvements in prognosis and survival can only come with protocols that will allow the detection and classification of prostate tumors as early as possible. Another potential source of biomarkers is exosomes. These nanovesicles measure $50-150 \mathrm{~nm}$ and are found in cells as early endosomes that contain miRNAs, mRNA, proteins, and lipids. Exosomes play a key role in intercellular communication and in the regulation of recipient cell biology, as well as in a range of physiological and pathological processes [112]. Some evidence now supports the idea that miRNA can be reliably sourced from blood-derived exosomes to identify disease biomarkers $[113,114]$.

In numerous tumor types, prognosis has been predicted based on exosomal miRNAs recovered from the blood [115]. The relevance of using the miRNAs found in exosomes to manage PCa was investigated in a few studies. Two of these studies have suggested that PCa could be differentiated based on miR-141 upregulation [116, 117]. Another study reported that $\mathrm{PCa}$ was associated with the upregulation of both miR-141 and miR-375 and that disease progression was correlated with the serum levels of these miRNAs [118]. A correlation has also been established between poor survival outcomes and plasma levels of exosomal miR-1290 and miR-375. Better predictions were achieved when these novel biomarkers were included in a clinical prognostic model [119, 120]. miR-574-3p, miR-141-5p, and miR-21$5 p$ were markedly upregulated in urinary exosomes from PCa patients extracted via lectin-based exosome agglutination [121]. By contrast, extraction of urinary exosomes by differential centrifugation revealed a marked increase only in the expression of miR-141. The presence of miR-19b was also examined in urinary exosomes extracted via differential centrifugation and revealed good potential for $\mathrm{PCa}$ detection [122].

Despite the inconsistencies in the reported results, the initial findings regarding exosomal biomarkers are encouraging. However, the determination of the usefulness of exosomes will first require standardization of the isolation and profiling methods and the performance of extensive clinical trials. Furthermore, miRNAs are not without their shortcomings and difficulties, just like other novel biomarkers for cancer. These shortcomings and difficulties stem from differences in how samples are selected, handled, processed, and prepared, as well as from disagreements regarding data normalization. Consequently, before miRNAs can be employed clinically, those shortcomings and difficulties must be addressed.

\section{Conclusion}

A great deal of inhomogeneity occurs in PCa. In some cases, the risk of disease progression is minimal, and the cancerspecific survival rates at a 15-year follow-up can be higher than $99 \%$. Around $50-60 \%$ of new diagnoses are low-risk $\mathrm{PCa}$ [123]. However, aggressive PCa is associated with a significant reduction in survival rate. Therefore, detection of the disease as early as possible is vital, as is the effective treatment of these cases. These advances can only come about with the development of better methods of early diagnosis to improve the survival rate.

A number of novel biomarkers related to PCa aggressiveness have been recently shown to have great clinical potential. The outcomes of prospective studies conducted in multiple centers have indicated that the use of the PHI and 4 Kscore biomarkers decrease the proportion of redundant biopsies in male individuals subjected to PSA screening. Various guidelines have advocated the use of these biomarkers $[124,125]$. Nevertheless, comparative analysis of the usefulness of biomarkers requires large-scale prospective studies and stringent control of the bias caused by patient preselection based on the levels of PSA in the serum.

Several miRNAs with possible oncogene or tumorsuppressing functions are deregulated in PCa. As revealed by miRNA profiling research, mRNAs can regulate gene transcription either on their own or in conjunction with other transcription factors, with the end result being disrupted cellular processes in PCa tissues [18, 125]. Novel 
approaches to PCa management could be identified by advances in the knowledge of the functions of miRNAs in controlling PCa development and progression [126, 127]. Research on the integrated function of biomarkers and combination with magnetic resonance imaging data is also worthwhile $[128,129]$. Comparative analyses are also needed on the outcomes achieved with blood biomarkers and the encouraging data yielded using PSA or PHI. It should be known that none single biomarker has the capacity to perfectly detect $\mathrm{PCa}$, and more detailed profiling of novel PCa biomarkers remains necessary.

\section{Conflicts of Interest}

The authors declare there is no conflict of interest.

\section{Acknowledgments}

This study was supported by the grant from the National Natural Science Foundation of China (Grant No. 81502208), and the grant funded by the Natural Science Foundation of Liaoning Province (Grant No. 20170541054).

\section{References}

[1] H. Gronberg, "Prostate cancer epidemiology," Lancet, vol. 361, no. 9360, pp. 859-864, 2003.

[2] M. Matsushita, K. Fujita, and N. Nonomura, "Influence of diet and nutrition on prostate cancer," International Journal of Molecular Sciences, vol. 21, no. 4, p. 1447, 2020.

[3] S. F. Peisch, E. L. Van Blarigan, J. M. Chan, M. J. Stampfer, and S. A. Kenfield, "Prostate cancer progression and mortality: a review of diet and lifestyle factors," World Journal of Urology, vol. 35, no. 6, pp. 867-874, 2017.

[4] S. Noh, E. Choi, C. H. Hwang, J. H. Jung, S. H. Kim, and B. Kim, "Dietary compounds for targeting prostate cancer," Nutrients, vol. 11, no. 10, p. 2401, 2019.

[5] K. S. Sfanos, S. Yegnasubramanian, W. G. Nelson, and A. M. De Marzo, "The inflammatory microenvironment and microbiome in prostate cancer development," Nature Reviews Urology, vol. 15, no. 1, pp. 11-24, 2018.

[6] A. M. De Marzo, E. A. Platz, S. Sutcliffe et al., "Inflammation in prostate carcinogenesis," Nature Reviews Cancer, vol. 7, no. 4, pp. 256-269, 2007.

[7] C. Della Pepa, C. Cavaliere, S. Rossetti et al., "Predictive comprehensive geriatric assessment in elderly prostate cancer patients: the prospective observational scoop trial results," Anti-Cancer Drugs, vol. 28, no. 1, pp. 104-109, 2017.

[8] A. Veccia, O. Caffo, U. de Giorgi et al., "Clinical outcomes in octogenarians treated with docetaxel as first-line chemotherapy for castration-resistant prostate cancer," Future Oncology, vol. 12, no. 4, pp. 493-502, 2016.

[9] R. D. Loberg, C. J. Logothetis, E. T. Keller, and K. J. Pienta, "Pathogenesis and treatment of prostate cancer bone metastases: targeting the lethal phenotype," Journal of Clinical Oncology, vol. 23, no. 32, pp. 8232-8241, 2005.

[10] M. Salagierski and J. A. Schalken, "Molecular diagnosis of prostate cancer: PCA3 and TMPRSS2: ERG gene fusion," The Journal of Urology, vol. 187, no. 3, pp. 795-801, 2012.

[11] R. J. Hendriks, I. M. van Oort, and J. A. Schalken, "Bloodbased and urinary prostate cancer biomarkers: a review and comparison of novel biomarkers for detection and treatment decisions," Prostate Cancer and Prostatic Diseases, vol. 20, no. 1, pp. 12-19, 2017.

[12] US Preventive Services Task Force, D. C. Grossman, S. J. Curry et al., "Screening for prostate cancer," Journal of the American Medical Association, vol. 319, no. 18, pp. 19011913, 2018.

[13] K. J. L. Bell, C. Del Mar, G. Wright, J. Dickinson, and P. Glasziou, "Prevalence of incidental prostate cancer: a systematic review of autopsy studies," International Journal of Cancer, vol. 137, no. 7, pp. 1749-1757, 2015.

[14] N. Sangster-Guity, B. Tu-Sekine, D. M. Raben, S. R. Denmeade, and S. A. Williams, "Mutational analysis of prostate-specific antigen defines the intrinsic proteolytic activity of the proPSA zymogen," The Prostate, vol. 76, no. 13, pp. 1203-1217, 2016.

[15] S. V. Carlsson and M. J. Roobol, "What's new in screening in 2015?," Current Opinion in Urology, vol. 26, no. 5, pp. 447458, 2016.

[16] X. Filella, "Towards personalized prostate cancer screening," Advances in Laboratory Medicine / Avances en Medicina de Laboratorio, vol. 1, no. 1, 2020.

[17] K. Fleshner, S. V. Carlsson, and M. J. Roobol, “The effect of the USPSTF PSA screening recommendation on prostate cancer incidence patterns in the USA," Nature Reviews Urology, vol. 14, no. 1, pp. 26-37, 2017.

[18] P. H. Carroll and J. L. Mohler, "NCCN guidelines updates: prostate cancer and prostate cancer early detection," Journal of the National Comprehensive Cancer Network, vol. 16, no. 5S, pp. 620-623, 2018.

[19] D. A. Sartori and D. W. Chan, "Biomarkers in prostate cancer: what's new?," Current Opinion in Oncology, vol. 26, no. 3, pp. 259-264, 2014.

[20] S. D. Mikolajczyk, L. S. Grauer, L. S. Millar et al., "A precursor form of PSA (pPSA) is a component of the free PSA in prostate cancer serum," Urology, vol. 50, no. 5, pp. 710-714, 1997.

[21] A. Lepor, W. J. Catalona, and S. Loeb, "The prostate health index: its utility in prostate cancer detection," The Urologic Clinics of North America, vol. 43, no. 1, pp. 1-6, 2016.

[22] X. Filella, L. Foj, J. M. Auge, R. Molina, and J. Alcover, "Clinical utility of \%p2PSA and prostate health index in the detection of prostate cancer," Clinical Chemistry and Laboratory Medicine, vol. 52, no. 9, pp. 1347-1355, 2014.

[23] C. Stephan, S. Vincendeau, A. Houlgatte, H. Cammann, K. Jung, and A. Semjonow, "Multicenter evaluation of [-2] proprostate-specific antigen and the prostate health index for detecting prostate cancer," Clinical Chemistry, vol. 59, no. 1, pp. 306-314, 2013.

[24] I. Heidegger and R. Pichler, "Re: Peter K.-F. Chiu, Chi-Fai $\mathrm{Ng}$, Axel Semjonow, et al. A multicentre evaluation of the role of the prostate health index (PHI) in regions with differing prevalence of prostate cancer: adjustment of PHI reference ranges is needed for European and Asian settings. Eur Urol 2019;75:558-61," European Urology, vol. 75, no. 6, pp. e158-e159, 2019.

[25] P. K.-F. Chiu, C.-F. Ng, A. Semjonow et al., "A multicentre evaluation of the role of the prostate health index (PHI) in regions with differing prevalence of prostate cancer: adjustment of PHI reference ranges is needed for European and Asian settings," European Urology, vol. 75, no. 4, pp. 558$561,2019$. 
[26] M. Lazzeri, A. Haese, A. de la Taille et al., "Serum isoform [-2]proPSA derivatives significantly improve prediction of prostate cancer at initial biopsy in a total PSA range of 2-10 ng/ml: a multicentric European study," European Urology, vol. 63, no. 6, pp. 986-994, 2013.

[27] S. Loeb, M. G. Sanda, D. L. Broyles et al., "The prostate health index selectively identifies clinically significant prostate cancer," The Journal of Urology, vol. 193, no. 4, pp. 1163-1169, 2015.

[28] W. Wang, M. Wang, L. Wang, T. S. Adams, Y. Tian, and J. Xu, "Diagnostic ability of \%p2PSA and prostate health index for aggressive prostate cancer: a meta-analysis," Scientific Reports, vol. 4, p. 5012, 2015.

[29] X. Filella and N. Gimenez, "Evaluation of [-2] pro PSA and prostate health index (phi) for the detection of prostate cancer: a systematic review and meta-analysis," Clinical Chemistry and Laboratory Medicine, vol. 51, pp. 729-739, 2012.

[30] D. Bruzzese, C. Mazzarella, M. Ferro et al., "Prostate health index vs percent free prostate-specific antigen for prostate cancer detection in men with "gray" prostate-specific antigen levels at first biopsy: systematic review and meta-analysis," Translational Research, vol. 164, no. 6, pp. 444-451, 2014.

[31] X. Filella, L. Foj, J. Alcover, J. M. Auge, R. Molina, and $\mathrm{W}$. Jimenez, "The influence of prostate volume in prostate health index performance in patients with total PSA lower than $10 \mu \mathrm{g} / \mathrm{L}$," Clinica Chimica Acta, vol. 436, pp. 303-307, 2014.

[32] I. Heidegger, H. Klocker, R. Pichler et al., "ProPSA and the prostate health index as predictive markers for aggressiveness in low-risk prostate cancer-results from an international multicenter study," Prostate Cancer and Prostatic Diseases, vol. 20, no. 3, pp. 271-275, 2017.

[33] C. de la Calle, D. Patil, J. T. Wei et al., "Multicenter evaluation of the prostate health index to detect aggressive prostate cancer in biopsy naïve men," The Journal of Urology, vol. 194, no. 1, pp. 65-72, 2015.

[34] J. J. Tosoian, S. Loeb, Z. Feng et al., "Association of [-2]proPSA with biopsy reclassification during active surveillance for prostate cancer," The Journal of Urology, vol. 188, no. 4, pp. 1131-1136, 2012.

[35] A. J. Vickers, A. M. Cronin, M. J. Roobol et al., "A fourkallikrein panel predicts prostate cancer in men with recent screening: data from the European Randomized Study of Screening for Prostate Cancer, Rotterdam," Clinical Cancer Research, vol. 16, no. 12, pp. 3232-3239, 2010.

[36] A. J. Vickers, A. M. Cronin, G. Aus et al., "Impact of recent screening on predicting the outcome of prostate cancer biopsy in men with elevated prostate-specific antigen: data from the European Randomized Study of Prostate Cancer Screening in Gothenburg, Sweden," Cancer, vol. 116, pp. 2612-2620, 2010.

[37] A. J. Vickers, A. Gupta, C. J. Savage et al., "A panel of kallikrein marker predicts prostate cancer in a large, population-based cohort followed for 15 years without screening," Cancer Epidemiology, Biomarkers \& Prevention, vol. 20, no. 2, pp. 255-261, 2011.

[38] S. V. Carlsson, M. T. Peltola, D. Sjoberg et al., "Can one blood draw replace transrectal ultrasonography-estimated prostate volume to predict prostate cancer risk?," BJU International, vol. 112, no. 5, pp. 602-609, 2013.

[39] T. Chongqing, P. Liubao, Z. Xiaohui et al., "Cost-utility analysis of the newly recommended adjuvant chemotherapy for resectable gastric cancer patients in the 2011 Chinese National Comprehensive Cancer Network (NCCN) clinical practice guidelines in oncology: gastric cancer," PharmacoEconomics, vol. 32, no. 3, pp. 235-243, 2014.

[40] A. Nirenberg, N. K. Reame, K. D. Cato, and E. L. Larson, "Oncology nurses' use of National Comprehensive Cancer Network clinical practice guidelines for chemotherapyinduced and febrile neutropenia," Oncology Nursing Forum, vol. 37, no. 6, pp. 765-773, 2010.

[41] D. J. Parekh, S. Punnen, D. D. Sjoberg et al., "A multiinstitutional prospective trial in the USA confirms that the 4Kscore accurately identifies men with high-grade prostate cancer," European Urology, vol. 68, no. 3, pp. 464-470, 2015.

[42] A. Vickers, E. A. Vertosick, D. D. Sjoberg et al., "Properties of the 4-kallikrein panel outside the diagnostic gray zone: metaanalysis of patients with positive digital rectal examination or prostate specific antigen $10 \mathrm{ng} / \mathrm{ml}$ and above," The Journal of Urology, vol. 197, 3 Part 1, pp. 607-613, 2017.

[43] R. J. Bryant, D. D. Sjoberg, A. J. Vickers et al., "Predicting high-grade cancer at ten-core prostate biopsy using four kallikrein markers measured in blood in the ProtecT study," Journal of the National Cancer Institute, vol. 107, no. 7, 2015.

[44] S. Carlsson, A. Maschino, F. Schroder et al., "Predictive value of four kallikrein markers for pathologically insignificant compared with aggressive prostate cancer in radical prostatectomy specimens: results from the European Randomized Study of Screening for Prostate Cancer section Rotterdam," European Urology, vol. 64, no. 5, pp. 693-699, 2013.

[45] A. Benchikh, C. Savage, A. Cronin et al., "A panel of kallikrein markers can predict outcome of prostate biopsy following clinical work-up: an independent validation study from the European Randomized Study of Prostate Cancer screening, France," BMC Cancer, vol. 10, no. 1, p. 635, 2010.

[46] A. J. Vickers, A. M. Cronin, G. Aus et al., "A panel of kallikrein markers can reduce unnecessary biopsy for prostate cancer: data from the European Randomized Study of Prostate Cancer Screening in Göteborg, Sweden," BMC Medicine, vol. 6, no. 1, p. 19, 2008.

[47] P. Stattin, A. J. Vickers, D. D. Sjoberg et al., "Improving the specificity of screening for lethal prostate cancer using prostate-specific antigen and a panel of kallikrein markers: a nested case-control study," European Urology, vol. 68, no. 2, pp. 207-213, 2015.

[48] T. Nordstrom, A. Vickers, M. Assel, H. Lilja, H. Gronberg, and M. Eklund, "Comparison between the four-kallikrein panel and prostate health index for predicting prostate cancer," European Urology, vol. 68, no. 1, pp. 139-146, 2015.

[49] P. V. Nazarov, S. E. Reinsbach, A. Muller et al., "Interplay of microRNAs, transcription factors and target genes: linking dynamic expression changes to function," Nucleic Acids Research, vol. 41, no. 5, pp. 2817-2831, 2013.

[50] X. Liu, Q. Chen, J. Yan et al., "MiRNA-296-3p-ICAM-1 axis promotes metastasis of prostate cancer by possible enhancing survival of natural killer cell-resistant circulating tumour cells," Cell Death \& Disease, vol. 4, no. 11, article e928, 2013.

[51] J. D. Arroyo, J. R. Chevillet, E. M. Kroh et al., "Argonaute2 complexes carry a population of circulating microRNAs independent of vesicles in human plasma," Proceedings of the National Academy of Sciences of the United States of America, vol. 108, no. 12, pp. 5003-5008, 2011.

[52] A. Gallo, M. Tandon, I. Alevizos, and G. G. Illei, “The majority of microRNAs detectable in serum and saliva is 
concentrated in exosomes," PLoS One, vol. 7, no. 3, article e30679, 2012.

[53] M. Jin, T. Zhang, C. Liu et al., "miRNA-128 suppresses prostate cancer by inhibiting BMI-1 to inhibit tumor-initiating cells," Cancer Research, vol. 74, no. 15, pp. 4183-4195, 2014.

[54] H. Lan, H. Lu, X. Wang, and H. Jin, "MicroRNAs as potential biomarkers in cancer: opportunities and challenges," BioMed Research International, vol. 2015, Article ID 125094, 17 pages, 2015.

[55] E. A. Hunt, D. Broyles, T. Head, and S. K. Deo, "MicroRNA detection: current technology and research strategies," Annual Review of Analytical Chemistry, vol. 8, no. 1, pp. 217-237, 2015.

[56] C. H. Lawrie, S. Gal, H. M. Dunlop et al., "Detection of elevated levels of tumour-associated microRNAs in serum of patients with diffuse large B-cell lymphoma," British Journal of Haematology, vol. 141, no. 5, pp. 672-675, 2008.

[57] J. Lu, G. Getz, E. A. Miska et al., "MicroRNA expression profiles classify human cancers," Nature, vol. 435, no. 7043, pp. 834-838, 2005.

[58] R. Sokilde, M. Vincent, A. K. Moller et al., "Efficient identification of miRNAs for classification of tumor origin," The Journal of Molecular Diagnostics, vol. 16, no. 1, pp. 106$115,2014$.

[59] S. Sakamoto, "Editorial comment to functional significance of aberrantly expressed microRNAs in prostate cancer," International Journal of Urology, vol. 22, no. 3, pp. 252-253, 2015.

[60] Y. Goto, A. Kurozumi, H. Enokida, T. Ichikawa, and N. Seki, "Functional significance of aberrantly expressed microRNAs in prostate cancer," International Journal of Urology, vol. 22, no. 3, pp. 242-252, 2015.

[61] X.-B. Shi, L. Xue, J. Yang et al., "An androgen-regulated miRNA suppresses Bak1 expression and induces androgenindependent growth of prostate cancer cells," Proceedings of the National Academy of Sciences of the United States of America, vol. 104, no. 50, pp. 19983-19988, 2007.

[62] A. Srivastava, H. Goldberger, A. Dimtchev et al., "MicroRNA profiling in prostate cancer - the diagnostic potential of urinary miR-205 and miR-214," PLoS One, vol. 8, no. 10, article e76994, 2013.

[63] S. Griffiths-Jones, R. J. Grocock, S. van Dongen, A. Bateman, and A. J. Enright, "miRBase: microRNA sequences, targets and gene nomenclature," Nucleic Acids Research, vol. 34, no. 90001, pp. D140-D144, 2006.

[64] G. A. Calin, C. D. Dumitru, M. Shimizu et al., "Nonlinear partial differential equations and applications: frequent deletions and down-regulation of micro- RNA genes miR15 and miR16 at 13q14 in chronic lymphocytic leukemia," Proceedings of the National Academy of Sciences of the United States of America, vol. 99, no. 24, pp. 1552415529, 2002.

[65] R. Kanwal, A. R. Plaga, X. Liu, G. C. Shukla, and S. Gupta, "MicroRNAs in prostate cancer: functional role as biomarkers," Cancer Letters, vol. 407, pp. 9-20, 2017.

[66] Y. Yang, J. X. Guo, and Z. Q. Shao, "miR-21 targets and inhibits tumor suppressor gene PTEN to promote prostate cancer cell proliferation and invasion: an experimental study," Asian Pacific Journal of Tropical Medicine, vol. 10, no. 1, pp. 87-91, 2017.

[67] S. E. Jalava, A. Urbanucci, L. Latonen et al., "Androgen-regulated miR-32 targets BTG2 and is overexpressed in castra- tion- resistant prostate cancer," Oncogene, vol. 31, no. 41, pp. 4460-4471, 2012.

[68] T. Li, R. S. Li, Y. H. Li et al., "miR-21 as an independent biochemical recurrence predictor and potential therapeutic target for prostate cancer," The Journal of Urology, vol. 187, no. 4, pp. 1466-1472, 2012.

[69] E. K. Amankwah, E. Anegbe, H. Park, J. Pow-Sang, A. Hakam, and J. Y. Park, "miR-21, miR-221 and miR-222 expression and prostate cancer recurrence among obese and non-obese cases," Asian Journal of Andrology, vol. 15, no. 2, pp. 226-230, 2013.

[70] A. Cannistraci, A. L. Di Pace, R. De Maria, and D. Bonci, "MicroRNA as new tools for prostate cancer risk assessment and therapeutic intervention: results from clinical data set and patients' samples," BioMed Research International, vol. 2014, Article ID 146170, 17 pages, 2014.

[71] U. G. Lo, C. F. Lee, M. S. Lee, and J. T. Hsieh, "The role and mechanism of epithelial-to-mesenchymal transition in prostate cancer progression," International Journal of Molecular Sciences, vol. 18, no. 10, p. 2079, 2017.

[72] U. G. Lo, D. Yang, and J. T. Hsieh, "The role of microRNAs in prostate cancer progression," Translational andrology and urology, vol. 2, no. 3, pp. 228-241, 2013.

[73] A. A. Moustafa, M. Ziada, A. Elshaikh et al., "Identification of micro RNA signature and potential pathway targets in prostate cancer," Experimental Biology and Medicine, vol. 242, pp. 536-546, 2016.

[74] G. L. Andriole, E. D. Crawford, R. L. Grubb et al., "Prostate cancer screening in the randomized prostate, lung, colorectal, and ovarian cancer screening trial: mortality results after 13 years of follow-up," Journal of the National Cancer Institute, vol. 104, no. 2, pp. 125-132, 2012.

[75] Z. Y. Abd Elmageed, Y. Yang, R. Thomas et al., "Neoplastic reprogramming of patient-derived adipose stem cells by prostate cancer cell-associated exosomes," Stem Cells, vol. 32, no. 4, pp. 983-997, 2014.

[76] X.-B. Shi, L. Xue, A. H. Ma, C. G. Tepper, H. J. Kung, and R. W. V. White, "miR-125b promotes growth of prostate cancer xenograft tumor through targeting proapoptotic genes," The Prostate, vol. 71, no. 5, pp. 538549, 2011.

[77] S. Amir, A. H. Ma, X. B. Shi, L. Xue, H. J. Kung, and R. W. Devere White, "Oncomir miR-125b suppresses p14ARF to modulate p53-Dependent and p53-independent apoptosis in prostate cancer," PLoS One, vol. 8, no. 4, article e61064, 2013.

[78] T. Sun, M. Yang, S. Chen et al., "The altered expression of miR-221/-222 and miR-23b/-27b is associated with the development of human castration resistant prostate cancer," The Prostate, vol. 72, no. 10, pp. 1093-1103, 2012.

[79] S. J. Seashols-Williams, W. Budd, G. C. Clark et al., "miR-9 acts as an oncomiR in prostate cancer through multiple pathways that drive tumour progression and metastasis," PLoS One, vol. 11, no. 7, article e0159601, 2016.

[80] T. I. Hsu, C. H. Hsu, K. H. Lee et al., "MicroRNA-18a is elevated in prostate cancer and promotes tumorigenesis through suppressing STK4 in vitro and in vivo," Oncogene, vol. 3, no. 4, article e99, 2014.

[81] G. Al-Kafaji, Z. T. Al-Naieb, and M. Bakhiet, "Increased oncogenic microRNA-18a expression in the peripheral blood of patients with prostate cancer: a potential novel non- 
invasive biomarker," Oncology Letters, vol. 11, no. 2, pp. 1201-1206, 2016.

[82] M. Norgaard, A. O. Jensen, J. B. Jacobsen, K. Cetin, J. P. Fryzek, and H. T. Sorensen, "Skeletal related events, bone metastasis and survival of prostate cancer: a population based cohort study in Denmark (1999 to 2007)," The Journal of Urology, vol. 184, no. 1, pp. 162-167, 2010.

[83] W. Lou, J. Liu, Y. Gao et al., "MicroRNAs in cancer metastasis and angiogenesis," Oncotarget, vol. 8, no. 70, pp. 115787115802, 2017.

[84] M. Gururajan, S. Josson, G. C. Chu et al., "miR-154* and miR379 in the DLK1-DIO3 microRNA mega-cluster regulate epithelial to mesenchymal transition and bone metastasis of prostate cancer," Clinical Cancer Research, vol. 20, no. 24, pp. 6559-6569, 2014.

[85] S. Josson, M. Gururajan, P. Hu et al., "miR-409-3p/-5p promotes tumorigenesis, epithelial-to-mesenchymal transition, and bone metastasis of human prostate cancer," Clinical Cancer Research, vol. 20, no. 17, pp. 4636-4646, 2014.

[86] M. K. Siu, Y. C. Tsai, Y. S. Chang et al., "Transforming growth factor- $\beta$ promotes prostate bone metastasis through induction of microRNA-96 and activation of the mTOR pathway," Oncogene, vol. 34, no. 36, pp. 4767-4776, 2015.

[87] D. Lin, F. Cui, Q. Bu, and C. Yan, "The expression and clinical significance of GTP-binding RAS-like 3(ARHI)and microRNA 221 and 222 in prostate cancer," The Journal of International Medical Research, vol. 39, no. 5, pp. 18701875, 2011.

[88] H. M. Lin, K. L. Mahon, C. Spielman et al., "Phase 2 study of circulating microRNA biomarkers in castration-resistant prostate cancer," British Journal of Cancer, vol. 116, no. 8, pp. 1002-1011, 2017.

[89] Y. Yang, D. Jia, H. Kim et al., "Dysregulation of miR-212 promotes castration resistance through hnRNPH1-mediated regulation of AR and AR-V7: implications for racial disparity of prostate cancer," Clinical Cancer Research, vol. 22, no. 7, pp. 1744-1756, 2016.

[90] E. R. Sherwood, J. L. Van Dongen, C. G. Wood, S. Liao, J. M. Kozlowski, and C. Lee, "Epidermal growth factor receptor activation in androgen-independent but not androgen-stimulated growth of human prostatic carcinoma cells," British Journal of Cancer, vol. 77, no. 6, pp. 855861, 1998.

[91] Q. Fu, X. Liu, Y. Liu, J. Yang, G. Lv, and S. Dong, "MicroRNA-335 and -543 suppress bone metastasis in prostate cancer via targeting endothelial nitric oxide synthase," International Journal of Molecular Medicine, vol. 36, no. 5, pp. 1417-1425, 2015.

[92] Q. Zhu, H. Li, Y. Li, and L. Jiang, "MicroRNA-30a functions as tumor suppressor and inhibits the proliferation and invasion of prostate cancer cells by down-regulation of SIX1," Human Cell, vol. 30, no. 4, pp. 290-299, 2017.

[93] D. Ren, M. Wang, W. Guo et al., "Double-negative feedback loop between ZEB2 and miR-145 regulates epithelialmesenchymal transition and stem cell properties in prostate cancer cells," Cell and Tissue Research, vol. 358, no. 3, pp. 763-778, 2014.

[94] Q. Chen, X. Zhao, H. Zhang et al., “MiR-130b suppresses prostate cancer metastasis through down-regulation of MMP2," Molecular Carcinogenesis, vol. 54, no. 11, pp. 12921300, 2015.
[95] C. P. Bracken, P. A. Gregory, Y. Khew-Goodall, and G. J. Goodall, "The role of microRNAs in metastasis and epithelial-mesenchymal transition," Cellular and Molecular Life Sciences, vol. 66, no. 10, pp. 1682-1699, 2009.

[96] J. Banyard, I. Chung, A. M. Wilson et al., "Regulation of epithelial plasticity by miR-424 and miR-200 in a new prostate cancer metastasis model," Scientific Reports, vol. 3, no. 1, p. 3151, 2013.

[97] J. H. Tseng, M. Bisogna, L. N. Hoang et al., "miR-200c-driven mesenchymal-to-epithelial transition is a therapeutic target in uterine carcinosarcomas," Scientific Reports, vol. 7, no. 1, p. 3614, 2017.

[98] J. Yu, Y. Lu, D. Cui et al., "miR-200b suppresses cell proliferation, migration and enhances chemosensitivity in prostate cancer by regulating Bmi-1," Oncology Reports, vol. 31, no. 2, pp. 910-918, 2014.

[99] P. Gandellini, V. Profumo, A. Casamichele et al., " $m i R-205$ regulates basement membrane deposition in human prostate: implications for cancer development," Cell Death and Differentiation, vol. 19, no. 11, pp. 1750-1760, 2012.

[100] B. Verdoodt, M. Neid, M. Vogt et al., "MicroRNA-205, a novel regulator of the anti-apoptotic protein $\mathrm{Bcl} 2$, is downregulated in prostate cancer," International Journal of Oncology, vol. 43, no. 1, pp. 307-314, 2013.

[101] B. Xu, N. Wang, X. Wang et al., "MiR-146a suppresses tumor growth and progression by targeting EGFR pathway and in a p-ERK-dependent manner in castration-resistant prostate cancer," The Prostate, vol. 72, no. 11, pp. 11711178, 2012.

[102] Y. N. Liu, J. Yin, B. Barrett et al., "Loss of androgen-regulated microRNA 1 activates SRC and promotes prostate cancer bone metastasis," Molecular and Cellular Biology, vol. 35, no. 11, pp. 1940-1951, 2015.

[103] M. K. Siu, W. Abou-Kheir, J. J. Yin et al., "Loss of EGFR signaling-regulated miR-203 promotes prostate cancer bone metastasis and tyrosine kinase inhibitors resistance," Oncotarget, vol. 5, no. 11, pp. 3770-3784, 2014.

[104] Z. Y. Lin, Y. Q. Huang, Y. Q. Zhang et al., "MicroRNA-224 inhibits progression of human prostate cancer by downregulating TRIB1," International Journal of Cancer, vol. 135, no. 3, pp. 541-550, 2014.

[105] K. Hanlon, C. E. Rudin, and L. W. Harries, "Investigating the targets of MIR-15a and MIR-16-1 in patients with chronic lymphocytic leukemia (CLL)," PLoS One, vol. 4, no. 9, p. e7169, 2009.

[106] W. Jin, F. Chen, K. Wang, Y. Song, X. Fei, and B. Wu, "miR$15 \mathrm{a} / \mathrm{miR}-16$ cluster inhibits invasion of prostate cancer cells by suppressing TGF- $\beta$ signaling pathway," Biomedicine \& Pharmacotherapy, vol. 104, pp. 637-644, 2018.

[107] R. I. Aqeilan, G. A. Calin, and C. M. Croce, “_miR-15a_ and _miR-16-1_ in cancer: discovery, function and future perspectives," Cell Death and Differentiation, vol. 17, no. 2, pp. 215-220, 2010.

[108] M. Musumeci, V. Coppola, A. Addario et al., "Control of tumor and microenvironment cross-talk by miR-15a and miR-16 in prostate cancer," Oncogene, vol. 30, no. 41, pp. 4231-4242, 2011.

[109] K. Sikand, J. E. Slaibi, R. Singh, S. D. Slane, and G. C. Shukla, "miR 488* inhibits androgen receptor expression in prostate carcinoma cells," International Journal of Cancer, vol. 129, no. 4, pp. 810-819, 2011. 
[110] A. Kroiss, S. Vincent, M. Decaussin-Petrucci et al., "Androgen-regulated microRNA-135a decreases prostate cancer cell migration and invasion through downregulating ROCK1 and ROCK2," Oncogene, vol. 34, no. 22, pp. 2846-2855, 2015.

[111] C. E. Fletcher, D. A. Dart, A. Sita-Lumsden, H. Cheng, P. S. Rennie, and C. L. Bevan, "Androgen-regulated processing of the oncomir miR-27a, which targets prohibitin in prostate cancer," Human Molecular Genetics, vol. 21, no. 14, pp. 3112-3127, 2012.

[112] A. Valentino, P. Reclusa, R. Sirera et al., "Exosomal microRNAs in liquid biopsies: future biomarkers for prostate cancer," Clinical \& Translational Oncology, vol. 19, no. 6, pp. 651-657, 2017.

[113] L. Cheng, R. A. Sharples, B. J. Scicluna, and A. F. Hill, "Exosomes provide a protective and enriched source of miRNA for biomarker profiling compared to intracellular and cellfree blood," Journal of Extracellular Vesicles, vol. 3, no. 1, 2014.

[114] L. Cheng, X. Sun, B. J. Scicluna, B. M. Coleman, and A. F. Hill, "Characterization and deep sequencing analysis of exosomal and non-exosomal miRNA in human urine," Kidney International, vol. 86, no. 2, pp. 433-444, 2014.

[115] D. C. Yu, Q. G. Li, X. W. Ding, and Y. T. Ding, "Circulating microRNAs: potential biomarkers for cancer," International Journal of Molecular Sciences, vol. 12, no. 3, pp. 2055-2063, 2011.

[116] P. S. Mitchell, R. K. Parkin, E. M. Kroh et al., "Circulating microRNAs as stable blood-based markers for cancer detection," Proceedings of the National Academy of Sciences of the United States of America, vol. 105, no. 30, pp. 10513-10518, 2008.

[117] M. J. Lodes, M. Caraballo, D. Suciu, S. Munro, A. Kumar, and B. Anderson, "Detection of cancer with serum miRNAs on an oligonucleotide microarray," PLoS One, vol. 4, no. 7, p. e6229, 2009.

[118] J. C. Brase, M. Johannes, T. Schlomm et al., "Circulating miRNAs are correlated with tumor progression in prostate cancer," International Journal of Cancer, vol. 128, no. 3, pp. 608-616, 2011.

[119] N. Mottet, J. Bellmunt, M. Bolla et al., "EAU-ESTRO-SIOG guidelines on prostate cancer. Part 1: screening, diagnosis, and local treatment with curative intent," European Urology, vol. 71, no. 4, pp. 618-629, 2017.

[120] N. Mottet, J. Bellmunt, M. Bolla et al., "EAU guidelines on prostate cancer. Part II: treatment of advanced, relapsing, and castration-resistant prostate cancer," European Urology, vol. 59, no. 4, pp. 572-583, 2011.

[121] R. Samsonov, T. Shtam, V. Burdakov et al., "Lectin-induced agglutination method of urinary exosomes isolation followed by mi-RNA analysis: application for prostate cancer diagnostic," The Prostate, vol. 76, no. 1, pp. 68-79, 2016.

[122] O. E. Bryzgunova, M. M. Zaripov, T. E. Skvortsova et al., "Comparative study of extracellular vesicles from the urine of healthy individuals and prostate cancer patients," PLoS One, vol. 11, no. 6, article e0157566, 2016.

[123] L. Klotz, "Low-risk prostate cancer can and should often be managed with active surveillance and selective delayed intervention," Nature Clinical Practice Urology, vol. 5, no. 1, pp. 2-3, 2008.

[124] A. J. Vickers, J. A. Eastham, P. T. Scardino, and H. Lilja, “The memorial Sloan Kettering cancer center recommendations for prostate cancer screening," Urology, vol. 91, pp. 12-18, 2016.

[125] M. C. Sighinolfi and B. Rocco, "Reply to Alessia Cimadamore, Marina Scarpelli, Liang Cheng, et al's Letter to the Editor, re: Maria Chiara Sighinolfi, Bernardo Rocco's Words of Wisdom re: EAU Guidelines: Prostate Cancer 2019. Mottet N, van den Bergh RCN, Briers E, et al. https://uroweb.org/guideline/prostate-Cancer/. Eur Urol 2019, 76:871," European Urology, vol. 77, no. 5, pp. e128-e129, 2020.

[126] S. C. Druskin, J. J. Tosoian, A. Young et al., "Combining Prostate Health Index density, magnetic resonance imaging and prior negative biopsy status to improve the detection of clinically significant prostate cancer," BJU International, vol. 121, no. 4, pp. 619-626, 2018.

[127] V. J. Gnanapragasam, K. Burling, A. George et al., "The prostate health index adds predictive value to multi-parametric MRI in detecting significant prostate cancers in a repeat biopsy population," Scientific Reports, vol. 6, no. 1, p. 35364, 2016.

[128] S. Washino, T. Okochi, K. Saito et al., "Combination of prostate imaging reporting and data system (PI-RADS) score and prostate-specific antigen (PSA) density predicts biopsy outcome in prostate biopsy naïve patients," BJU International, vol. 119, no. 2, pp. 225-233, 2017.

[129] P. J. L. De Visschere, A. Briganti, J. J. Fütterer et al., "Role of multiparametric magnetic resonance imaging in early detection of prostate cancer," Insights into Imaging, vol. 7, no. 2, pp. 205-214, 2016. 